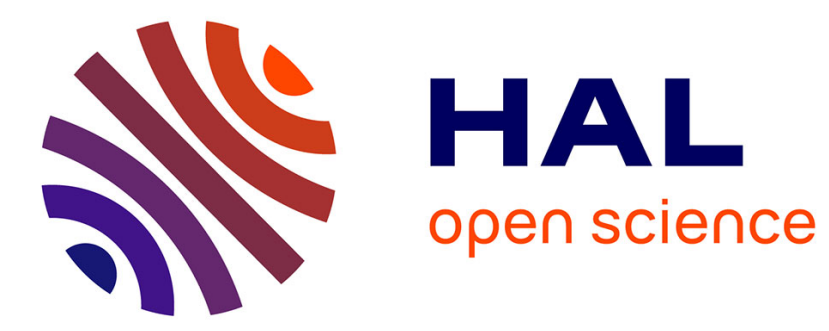

\title{
Sur les processus linéaires de naissance et de mort dans un environnement aléatoire
}

\author{
Nicolas Bacaër, Abdelkarim Ed-Darraz
}

\section{To cite this version:}

Nicolas Bacaër, Abdelkarim Ed-Darraz. Sur les processus linéaires de naissance et de mort dans un environnement aléatoire. 2014. hal-01266287v3

\section{HAL Id: hal-01266287 \\ https://hal.science/hal-01266287v3}

Submitted on 24 May 2020

HAL is a multi-disciplinary open access archive for the deposit and dissemination of scientific research documents, whether they are published or not. The documents may come from teaching and research institutions in France or abroad, or from public or private research centers.
L'archive ouverte pluridisciplinaire HAL, est destinée au dépôt et à la diffusion de documents scientifiques de niveau recherche, publiés ou non, émanant des établissements d'enseignement et de recherche français ou étrangers, des laboratoires publics ou privés. 


\title{
Sur les processus linéaires de naissance et de mort dans un environnement aléatoire
}

\author{
Nicolas Bacaër* Abdelkarim Ed-Darraz ${ }^{\dagger}$ \\ J. Math. Biol. 69 (2014) 73-90 \\ hal : 01266287 \\ Traductions : [ar, de, es, it, ja, nl, pt, ru, zh], [html]
}

\begin{abstract}
Résumé
On étudie la probabilité d'extinction pour des processus linéaires de naissance et de mort à un ou plusieurs types dans un environnement évoluant suivant une chaîne de Markov. La probabilité d'extinction est égale à 1 presque sûrement si et seulement si la reproductivité nette $R_{0}$ est inférieure ou égale à 1 , le point clé étant d'identifier la définition convenable de $R_{0}$ telle que ce résultat soit vrai.
\end{abstract}

\section{Introduction}

Un article récent de Bacaër et Ait Dads (2012) étudie la probabilité d'extinction $\omega$ pour un processus linéaire de naissance et de mort avec plusieurs types dans un environnement périodique. Dans ce cas $\omega=1$ si un certain nombre, appelé reproductivité nette et noté $R_{0}$ suivant Dublin et Lotka (1925), est inférieur ou égal à 1 , tandis que $\omega<1$ si $R_{0}>1$. L'emphase mise sur le paramètre seuil $R_{0}$ est motivée par des applications en épidémiologie. La preuve utilise la technique standard (Kendall, 1948) basée sur l'équation aux dérivées partielles linéaire du premier ordre satisfaite par une fonction génératrice. Pour les modèles à un seul type avec un taux de naissance $a(t)$ et une mortalité $b(t) T$ périodiques, on a $R_{0}=\left(\int_{0}^{T} a(t) d t\right) /\left(\int_{0}^{T} b(t) d t\right)$. Le même $R_{0}$ sert aussi de seuil pour les modèles de population périodiques sans stochasticité démographique (Bacaër et Guernaoui, 2006, Sect. 5).

L'objectif ici est d'étudier les processus linéaires de naissance et de mort à plusieurs types dans un environnement aléatoire. Essayons de résumer la littérature sur ce sujet. Pour des modèles de population en temps discret dans un

\footnotetext{
*Institut de Recherche pour le Développement, Bondy, France. Courriel : nicolas.bacaer@ird.fr

${ }^{\dagger}$ Université Cadi Ayyad, Département de mathématiques, Marrakech, Maroc.
} 
environnement aléatoire mais sans stochasticité démographique, Lewontin et Cohen (1969) ont remarqué que l'espérance de la population peut croître à l'infini même si l'extinction se produit presque sûrement; voir aussi Haccou et al. (2005). Athreva et Karlin (1971) ont étudié les processus de branchement en temps discret dans un environnement aléatoire, à la fois dans le cas d'un seul type et celui de plusieurs types. Cogburn et Torrez (1981) ont étudié les processus linéaires de naissance et de mort à un seul type dans un environnement aléatoire. Pour le cas particulier d'un nombre fini d'environnements, soient $a_{k}$ le taux de naissance et $b_{k}$ la mortalité dans l'environnement $k$. Soit $u_{k}$ la proportion moyenne du temps passée dans l'environnement $k$. Leur corollaire 3.2 suggère que $\omega=1$ si et seulement si

$$
\sum_{k}\left(a_{k}-b_{k}\right) u_{k} \leq 0 .
$$

Leur preuve est basée sur des résultats dus à Kaplan (1973). Plus récemment, Britton et Lindholm (2009) ont étudié le même processus à un seul type dans le cas particulier de deux environnements. Ils ont suggéré que $\omega=1$ si et seulement si $R_{\star} \leq 1$, où $R_{\star}=m_{1} m_{2}, m_{k}=\int_{0}^{\infty} q_{k} e^{-q_{k} \tau} e^{\left(a_{k}-b_{k}\right) \tau} d \tau$ et $q_{k}$ est la taux auquel l'environnement quitte l'état $k$. Ils ont aussi montré que $R_{\star}$ avait la même position par rapport à 1 que le rayon spectral de la « matrice de prochaine génération »

$$
\left(\begin{array}{cc}
a_{1} & 0 \\
0 & a_{2}
\end{array}\right)\left(\begin{array}{cc}
b_{1}+q_{1} & -q_{2} \\
-q_{1} & b_{2}+q_{2}
\end{array}\right)^{-1}
$$

rayon spectral qu'ils ont appelé $\ll R_{0} \gg$. Gray et al. (2012) ont étudié un modèle de population en temps continu et environnement aléatoire mais sans stochasticité démographique. Ils ont remarqué que la position de

$$
\frac{\sum_{k} a_{k} u_{k}}{\sum_{k} b_{k} u_{k}}
$$

par rapport à 1 sert de seuil pour l'extinction mais ont appelé ce nombre $T_{0}$, réservant la notation $\ll R_{0} \gg$ pour le rayon spectral de (2). Remarquer que le seuil donné par (3) est le même que (10). Bacaër et Khaladi (2012) ont montré que (3) était le rayon spectral d'un opérateur « de prochaine géneration $\gg$ en dimension infinie et ont suggéré de garder la notation $R_{0}$ pour ce nombre. Hernandez-Suarez et al. (2012) et Artalejo et al. (2012) ont également abordé des problèmes reliés concernant $R_{0}$. Comme on le verra ci-dessous, à part le problème des notations, (3) et $R_{\star}$ (ou le rayon spectral de (2)) peuvent ne pas avoir la même position par rapport à 1 .

Dans la section 2, on présente deux preuves alternatives du résultat de Cogburn et Torrez (1981) pour les modèles à un seul type. La première preuve utilise une formule de Kendall (1948) pour la probabilité d'extinction dans un environnement variable. La seconde preuve ramène le problème en temps continu au cadre en temps discret d Athreva et Karlin (1971). On discute aussi en détail un exemple simple avec juste deux environnements, qui espérons-le clarifiera les problèmes concernant la définition de $R_{0}$ mentionnés ci-dessus. La section 3 
étudie la probabilité d'extinction pour des populations de plusieurs types dans un cadre avec temps continu. On réduit une nouvelle fois le problème au cas en temps discret d Athreya et Karlin (1971). Ceci ne semble possible que pour un nombre fini d'environnements. Il se peut que l'approche suivant Kendall (1948) puisse aussi être généralisée au cas de plusieurs types en utilisant les résultats de Chueshov (2002) ou de Benaïm et Schreiber (2009), éventuellement sans la restriction d'un nombre fini d'environnements. On présente aussi des simulations numériques. La conclusion suggère d'autres possibilités de généralisation.

\section{Le modèle à un seul type}

\subsection{La probabilité d'extinction}

Soit un processus linéaire de naissance et de mort à un seul type dans un environnement variable. Soit $a(t)$ le taux de naissance et $b(t)$ la mortalité au temps $t$. Supposons que $a(t)=a_{\theta(t)}$ et $b(t)=b_{\theta(t)}$, où $\theta(t)$ est un processus stochastique à valeurs dans $\{1,2, \ldots, K\}$ représentant différents environnements. Supposons que $a_{k}>0$ et $b_{k}>0$ pour tout $k$. Supposons que les basculements entre les environnements suivent une chaîne de Markov homogène en temps continu. Pour $k \neq \ell$, soit $Q_{k, \ell} \geq 0$ le taux auquel l'environnement bascule de $\ell$ vers $k$. Soit $Q$ la matrice correspondante avec $Q_{\ell, \ell}=-q_{\ell}$ et $q_{\ell}=\sum_{k \neq \ell} Q_{k, \ell}$. Supposons que la matrice $Q$ soit irréductible. Ceci implique que $q_{k}>0$ pour tout $k$. Par conséquent, il existe une unique distribution de probabilités stationnaire et strictement positive $u$ telle que $Q u=0, \sum_{k} u_{k}=1$ et $u_{k}>0$ pour tout $k$ (Pardoux, 2008, p. 147). Soit $R_{0}$ donné par la formule (3). Soit $\omega$ la probabilité d'extinction partant d'un individu au temps 0 dans l'environnement $k_{0}$. La proposition suivante, bien qu'un cas spécial des résultats de Cogburn et Torrez (1981), sera démontrée d'une manière un peu plus simple de deux façons différentes.

Proposition 1. Si $R_{0} \leq 1$, alors $\omega=1$ presque sûrement. Si $R_{0}>1$, alors $\omega<1$ presque sûrement.

Démonstration. On sait d'après Kendall (1948, Eq. (18)) que

$$
\omega=1-\frac{1}{1+\int_{0}^{\infty} b(s) \exp \left[\int_{0}^{s}(b(v)-a(v)) d v\right] d s},
$$

que l'intégrale au dénominateur soit finie ou infinie. Le théorème ergodique (Pardoux, 2008, p. 150) montre que

$$
\lim _{s \rightarrow+\infty} \frac{1}{s} \int_{0}^{s}(b(v)-a(v)) d v=\sum_{k}\left(b_{k}-a_{k}\right) u_{k}
$$

presque sûrement. Si $R_{0}<1$, alors $\sum_{k}\left(b_{k}-a_{k}\right) u_{k}>0$ et l'intégrale au dénominateur de (4) diverge. Donc $\omega=1$. Si $R_{0}>1$, alors $\sum_{k}\left(b_{k}-a_{k}\right) u_{k}<0$ et l'intégrale au dénominateur de (4) converge. Donc $\omega<1$. 
On n'a pas considéré le cas critique $R_{0}=1$. Cependant, voici une seconde preuve de la proposition 1 qui couvre aussi le cas critique.

Démonstration. Le processus environnemental en temps continu à $K$ états peut être vu comme une chaîne de Markov en temps discret sur l'espace d'états $\mathcal{X}=\{1,2, \ldots, K\} \times \mathbb{R}_{+}$, chaque pas de temps correspondant au temps entre deux basculements de l'environnement. Au lieu de dire par exemple que l'environnement est dans l'état $k$ pour $t$ unités de temps puis dans l'état $k^{\prime}$ pour $t^{\prime}$ unités de temps (avec $k^{\prime} \neq k$ ), on dit que l'état $(k, t) \in \mathcal{X}$ est suivi de l'état $\left(k^{\prime}, t^{\prime}\right) \in \mathcal{X}$. La probabilité pour que l'environnement $k$ soit suivi par l'environnement $k^{\prime}$ est

$$
\Pi_{k^{\prime}, k}=\left\{\begin{array}{lll}
\frac{Q_{k^{\prime}, k}}{q_{k}} & \text { si } & k^{\prime} \neq k \\
0 & \text { si } & k^{\prime}=k .
\end{array}\right.
$$

L'environnement $k^{\prime}$ dure entre $t^{\prime}$ et $t^{\prime}+d t^{\prime}$ unités de temps ( $d t^{\prime}$ infiniment petit) avec une probabilité $q_{k^{\prime}} e^{-q_{k^{\prime}} t^{\prime}} d t^{\prime}$. Donc la probabilité pour que l'environnement $k$ durant $t$ unités de temps soit suivi par l'environnement $k^{\prime}$ durant entre $t^{\prime}$ et $t^{\prime}+d t^{\prime}$ unités de temps est

$$
P_{(k, t) \rightarrow\left(k^{\prime}, t^{\prime}\right)} d t^{\prime}=\Pi_{k^{\prime}, k} q_{k^{\prime}} e^{-q_{k^{\prime}} t^{\prime}} d t^{\prime} .
$$

Remarquer que

$$
\int_{0}^{\infty} \sum_{k^{\prime}} P_{(k, t) \rightarrow\left(k^{\prime}, t^{\prime}\right)} d t^{\prime}=1 .
$$

Plus généralement, pour $z \in\{1,2, \ldots\}$, la probabilité de transition en $z$ étapes est donnée par

$$
P_{(k, t) \rightarrow\left(k^{\prime}, t^{\prime}\right)}^{(z)} d t^{\prime}=\left(\Pi^{z}\right)_{k^{\prime}, k} q_{k^{\prime}} e^{-q_{k^{\prime}} t^{\prime}} d t^{\prime},
$$

où $\Pi^{z}$ est la $z^{\mathrm{e}}$ puissance de la matrice $\Pi=\left(\Pi_{k^{\prime}, k}\right)$. Comme $Q u=0$ est équivalent à $\sum_{k \neq k^{\prime}} Q_{k^{\prime}, k} u_{k}=q_{k^{\prime}} u_{k^{\prime}}$ pour tout $k^{\prime}$, on peut vérifier que

$$
w_{k, t}=\frac{q_{k} u_{k}}{\sum_{\ell} q_{\ell} u_{\ell}} q_{k} e^{-q_{k} t}
$$

vérifie

$$
\sum_{k} \int_{0}^{\infty} w_{k, t} d t=1 \quad \text { et } \quad \sum_{k} \int_{0}^{\infty} w_{k, t} P_{(k, t) \rightarrow\left(k^{\prime}, t^{\prime}\right)} d t=w_{k^{\prime}, t^{\prime}}
$$

pour tout $\left(k^{\prime}, t^{\prime}\right) \in \mathcal{X}$. Donc $\left(w_{k, t}\right)$ est une distribution de probabilités stationnaire et la chaîne de Markov sur $\mathcal{X}$ est récurrente positive (Mevn et Tweedie, 1993). Athreva et Karlin (1971) mentionnent dans une remarque suivant leur théorème 4 que leurs résultats restent valables non seulement pour les chaînes de Markov irréductibles récurrentes positives sur un espace d'états dénombrable mais aussi pour un processus stationnaire ergodique sur un espace d'états général (non dénombrable) tel que $\mathcal{X}$.

Entre les basculements, on a un processus linéaire de naissance et de mort dans un environnement constant. Dans un environnement $k$ durant $t$ unités de 
temps, soit $\phi_{k, t}(x)$ la fonction génératrice de la population au bout de l'intervalle de temps, partant d'un individu au début de cet intervalle de temps :

$$
\phi_{k, t}(x)=\frac{b_{k}(1-x) e^{t\left(a_{k}-b_{k}\right)}+a_{k} x-b_{k}}{a_{k}(1-x) e^{t\left(a_{k}-b_{k}\right)}+a_{k} x-b_{k}}
$$

si $a_{k} \neq b_{k}$ tandis que

$$
\phi_{k, t}(x)=\frac{x+(1-x) a_{k} t}{1+(1-x) a_{k} t}
$$

si $a_{k}=b_{k}$ (Hillion, 1986, p. 118). L'espérance de la population au bout de l'intervalle de temps est égale à $\phi_{k, t}^{\prime}(1)=e^{\left(a_{k}-b_{k}\right) t}$. La probabilité que la population soit éteinte au bout de l'intervalle de temps est

$$
\phi_{k, t}(0)=\frac{1-e^{\left(a_{k}-b_{k}\right) t}}{1-e^{\left(a_{k}-b_{k}\right) t} a_{k} / b_{k}}
$$

si $a_{k} \neq b_{k}$, tandis que $\phi_{k, t}(0)=a_{k} t /\left(1+a_{k} t\right)$ si $a_{k}=b_{k}$. Si $a_{k}<b_{k}$, alors $1-\phi_{k, t}(0) \sim\left(1-a_{k} / b_{k}\right) e^{\left(a_{k}-b_{k}\right) t}$ quand $t \rightarrow+\infty$. Si $a_{k}=b_{k}$, alors $1-\phi_{k, t}(0) \sim$ $1 /\left(a_{k} t\right)$ quand $t \rightarrow+\infty$. Donc on peut facilement vérifier que

$$
\begin{aligned}
& \mathbb{E}(|\log (1-\phi(0))|)=\sum_{k} \int_{0}^{\infty} w_{k, t}\left|\log \left(1-\phi_{k, t}(0)\right)\right| d t<+\infty, \\
& \mathbb{E}\left(\left[\log \phi^{\prime}(1)\right]^{+}\right)=\sum_{k} \int_{0}^{\infty} w_{k, t}\left[\log \phi_{k, t}^{\prime}(1)\right]^{+} d t<+\infty,
\end{aligned}
$$

à cause de la décroissance exponentielle de $w_{k, t}$ par rapport à $t$. Avec Athreya et Karlin (1971), on conclut que $\omega=1$ si et seulement si

$$
\mathbb{E}\left(\log \phi^{\prime}(1)\right)=\sum_{k} \int_{0}^{\infty} w_{k, t} \log \phi_{k, t}^{\prime}(1) d t \leq 0
$$

Comme $\int_{0}^{\infty} t e^{-q_{k} t} d t=1 /\left(q_{k}\right)^{2}$, on obtient

$$
\mathbb{E}\left(\log \phi^{\prime}(1)\right)=\sum_{k} \int_{0}^{\infty} \frac{q_{k} u_{k}}{\sum_{\ell} q_{\ell} u_{\ell}} q_{k} e^{-q_{k} t}\left[\left(a_{k}-b_{k}\right) t\right] d t=\frac{\sum_{k}\left(a_{k}-b_{k}\right) u_{k}}{\sum_{\ell} q_{\ell} u_{\ell}}
$$

Donc $\omega=1$ si et seulement si $R_{0} \leq 1$.

\subsection{Un exemple et quelques remarques}

Comme dans l'exemple de Britton et Lindholm (2009, section 3), supposons qu'il y ait deux environnements : $a_{1}=2,7$ et $b_{1}=2$ d'une part, $a_{2}=0,8$ et $b_{2}=2$ d'autre part. Supposons que la matrice $Q$ soit

$$
Q=\left(\begin{array}{cc}
-q_{1} & q_{2} \\
q_{1} & -q_{2}
\end{array}\right)
$$



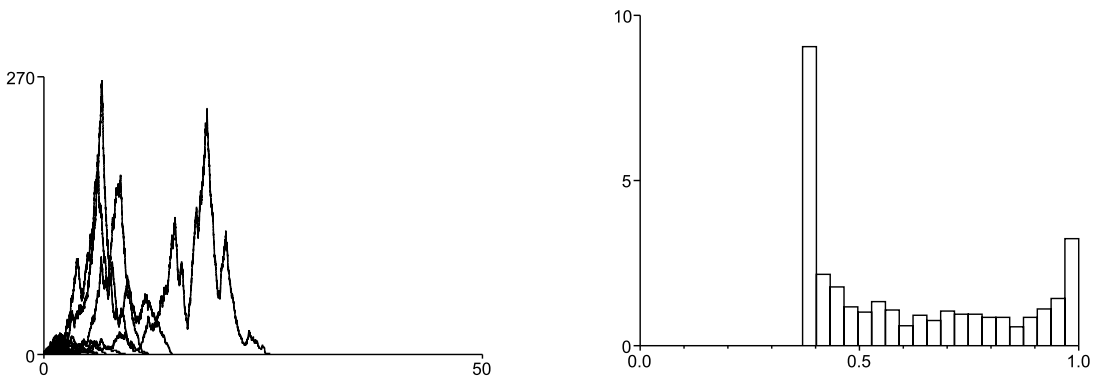

FIgURE 1 - À gauche (figure 1a) : 100 simulations de la population en fonction du temps $t$ dans le cas où $a_{1}=2,7$, partant d'un individu dans l'environnement 1 ; toutes les simulations conduisent à l'extinction. À droite (figure 1b) : partant d'un individu dans l'environnement 1 mais avec $a_{1}=5,4$, on a simulé 1000 histoires pour l'environnement et calculé la probabilité d'extinction $\omega$ par la formule (44) ; la figure montre un histogramme des valeurs prises par $\omega(0 \leq \omega \leq$ $1)$.

avec $q_{1}=q_{2}=1$. Alors $u_{1}=q_{2} /\left(q_{1}+q_{2}\right)=0,5, u_{2}=q_{1} /\left(q_{1}+q_{2}\right)=0,5$ et $R_{0}=$ $0,875<1$. Donc $\omega=1$. Des simulations numériques tendent à confirmer cette conclusion (figure 1a).

Comme autre exemple, considérons les mêmes valeurs des paramètres sauf que $a_{1}$ est doublé : $a_{1}=5,4$. Dans ce cas on a $R_{0}=1,55>1$. La figure $1 \mathrm{~b}$ montre un histogramme de la probabilité d'extinction $\omega$ partant d'une personne dans l'environnement 1. La moyenne de la probabilité d'extinction est environ 0,61 (elle serait 0,85 partant de l'environnement 2). L'histogramme a été obtenu en approchant la chaîne de Markov en temps continu gouvernant l'environnement pour $0<t<100$ par une chaîne de Markov en temps discret avec un pas de temps $\varepsilon=0,00005$. L'ordinateur choisit aléatoirement 1000 réalisations de cette chaîne de Markov, formant 1000 histoires environnementales. La formule (44) pour $\omega$ est ensuite estimée numériquement. Vu que $b_{1}=b_{2}, a_{2} \leq a_{1}$ et $a_{1} \geq b_{1}$, on s'aperçoit facilement avec (4) que $\omega \geq b_{1} / a_{1}$, cette borne inférieure correspondant à la probabilité d'extinction si l'environnement est toujours 1 . Avec les valeurs numériques ci-dessus, on obtient $\omega \geq 2 / 5,4 \simeq 0,37$, en accord avec la figure $1 b$.

Sous-criticalité du modèle discrétisé. Pour tracer la figure 1, on a discrétisé le processus en temps continu en utilisant un pas de temps $\varepsilon>0$. Pour simplifier, supposons comme dans l'exemple qu'il n'y ait que $K=2$ environnements. La matrice de transition, stochastique par colonnes, de la chaîne de Markov en temps discret est

$$
\mathcal{P}=\left(\begin{array}{cc}
1-q_{1} \varepsilon & q_{2} \varepsilon \\
q_{1} \varepsilon & 1-q_{2} \varepsilon
\end{array}\right) .
$$


Sa distribution stationnaire $\varpi$ est telle que $\mathcal{P} \varpi=\varpi$ avec $\sum \varpi_{k}=1$. On obtient $\varpi_{1}=q_{2} /\left(q_{1}+q_{2}\right)$ et $\varpi_{2}=q_{1} /\left(q_{1}+q_{2}\right)$, qui sont indépendants de $\varepsilon$ et coïncident avec les distributions stationnaires $u_{1}$ et $u_{2}$ du processus en temps continu. Si l'environnement est de type $k(k=1$ ou 2$)$, on a supposé que pendant un pas de temps chaque individu a une probabilité $a_{k} \varepsilon$ de donner naissance et une probabilité $b_{k} \varepsilon$ de mourir. Donc chaque individu conduit à 0 individu au pas de temps suivant avec une probabilité $\left(1-a_{k} \varepsilon\right) b_{k} \varepsilon$ [pas de naissance, une mort], à 1 individu avec une probabilité $\left(1-a_{k} \varepsilon\right)\left(1-b_{k} \varepsilon\right)+a_{k} \varepsilon b_{k} \varepsilon$ [soit pas de naissance et pas de mort, soit une naissance et une mort], et à 2 individus avec une probabilité $a_{k} \varepsilon\left(1-b_{k} \varepsilon\right)$ [une naissance, pas de mort]. La moyenne de cette distribution est $1+a_{k} \varepsilon-b_{k} \varepsilon$. Donc suivant la théorie des processus de branchement en temps discret en environnement aléatoire (Athreva et Karlin, 1971), le processus est sous-critique et conduit à l'extinction presque sûrement si et seulement si le paramètre seuil

$$
T(\varepsilon)=\sum_{k} \varpi_{k} \log \left(1+\left(a_{k}-b_{k}\right) \varepsilon\right)
$$

est négatif ou nul. Rappelons que $\varpi_{k}=u_{k}$. Lorsque $\varepsilon \rightarrow 0$, on voit que $T(\varepsilon) \sim$ $\varepsilon \sum_{k} u_{k}\left(a_{k}-b_{k}\right)$. Cette expression a le même signe que $R_{0}-1$.

Une autre manière d'utiliser les résultats en temps discret d'Athreya et Karlin. Une autre manière de voir cet exemple avec seulement deux environnements est de le considérer comme un processus de branchement dans une suite « d'environnements $\gg$ aléatoires indépendants et identiquement distribués (i.i.d.). En effet la suite d'environnements $1 \rightarrow 2$ se répète à l'identique, le temps passé dans chaque environnement étant aléatoire. La probabilité pour que l'environnement $k(k=1$ ou 2$)$ dure entre $t_{k}$ et $t_{k}+d t_{k}$ unités de temps est $q_{k} e^{-q_{k} t_{k}} d t_{k}$; ces probabilités sont indépendantes. Le nouvel espace des « environnements $\gg$ est donc $\left\{\left(t_{1}, t_{2}\right) \in\left(\mathbb{R}_{+}\right)^{2}\right\}$. La croissance moyenne durant une séquence $1 \rightarrow 2$ sachant que chaque environnement dure $t_{k}$ unités de temps est $\mathcal{M}=e^{\left(a_{1}-b_{1}\right) t_{1}} e^{\left(a_{2}-b_{2}\right) t_{2}}$. La théorie des processus de branchement dans les environnements i.i.d. (Athreya et Karlin, 1971) montre que $\omega=1$ si et seulement si

$$
\mathbb{E}(\log \mathcal{M})=\int_{0}^{\infty} \int_{0}^{\infty} q_{1} e^{-q_{1} t_{1}} q_{2} e^{-q_{2} t_{2}} \log \left(e^{\left(a_{1}-b_{1}\right) t_{1}} e^{\left(a_{2}-b_{2}\right) t_{2}}\right) d t_{1} d t_{2} \leq 0 .
$$

Mais on voit que

$$
\begin{aligned}
\mathbb{E}(\log \mathcal{M}) & =\int_{0}^{\infty} \int_{0}^{\infty} q_{1} e^{-q_{1} t_{1}} q_{2} e^{-q_{2} t_{2}}\left[\left(a_{1}-b_{1}\right) t_{1}+\left(a_{2}-b_{2}\right) t_{2}\right] d t_{1} d t_{2} \\
& =\int_{0}^{\infty} q_{1} e^{-q_{1} t_{1}}\left(a_{1}-b_{1}\right) t_{1} d t_{1}+\int_{0}^{\infty} q_{2} e^{-q_{2} t_{2}}\left(a_{2}-b_{2}\right) t_{2} d t_{2} .
\end{aligned}
$$

Donc

$$
\mathbb{E}(\log \mathcal{M})=\frac{\left(a_{1}-b_{1}\right) q_{2}+\left(a_{2}-b_{2}\right) q_{1}}{q_{1} q_{2}} .
$$


Le signe de cette quantité est le même que celui de $R_{0}-1$. Il y a extinction presque sûre si et seulement si $R_{0} \leq 1$.

Une autre manière de calculer la probabilité d'extinction. Soit $p_{k, n}(t)$ la probabilité que la population soit dans l'environnement $k$ au temps $t$ avec $n$ individus. Le processus est considéré comme une chaîne de Markov homogène en temps continu sur l'ensemble $\{1,2, \ldots, K\} \times \mathbb{N}$. Comme dans l'équation (2) de Yechiali (1973), on obtient

$$
\begin{aligned}
\frac{d p_{k, n}}{d t}= & -\left(a_{k}+b_{k}\right) n p_{k, n}+b_{k}(n+1) p_{k, n+1}+a_{k}(n-1) p_{k, n-1} \\
& +\sum_{\ell \neq k}\left(Q_{k, \ell} p_{\ell, n}-Q_{\ell, k} p_{k, n}\right)
\end{aligned}
$$

Considérons la chaîne de Markov en temps discret induite, obtenue en ne considérant que les sauts du processus en temps continu. Une fois dans l'état $(k, n)$, il y a une probabilité $a_{k} n /\left(a_{k} n+b_{k} n+q_{k}\right)$ de sauter vers l'état $(k, n+1)$, une probabilité $b_{k} n /\left(a_{k} n+b_{k} n+q_{k}\right)$ de sauter vers l'état $(k, n-1)$ et une probabilité $Q_{\ell, k} /\left(a_{k} n+b_{k} n+q_{k}\right)$ de sauter vers l'état $(\ell, n)$ pour tout $\ell \neq k$. Ordonnons les états $(k, n)$ ainsi : $(1,0), \ldots,(K, 0),(1,1), \ldots,(K, 1)$, etc. Soit $\pi_{k, n}(j)$ la probabilité d'être dans l'état $(k, n)$ après $j$ sauts. Soit $\pi(j)=\left(\pi_{k, n}(j)\right)$ le vecteur formé de ces probabilités, avec les indices $(k, n)$ ordonnés comme cidessus. Posons $\delta_{k, \ell}=1$ si $k=\ell$ et $\delta_{k, \ell}=0$ si $k \neq \ell$ : c'est le symbole de Kronecker. Pour tout $n$ (avec $n \geq 0$ ou $n \geq 1$ ) et tout $1 \leq k, \ell \leq K$, posons

$$
\begin{gathered}
L_{k, \ell}^{(n)}=\frac{(n-1) a_{\ell} \delta_{k, \ell}}{(n-1)\left(a_{\ell}+b_{\ell}\right)+q_{\ell}}, \quad M_{k, \ell}^{(n)}=\frac{Q_{k, \ell}\left(1-\delta_{k, \ell}\right)}{n\left(a_{\ell}+b_{\ell}\right)+q_{\ell}}, \\
N_{k, \ell}^{(n)}=\frac{(n+1) b_{\ell} \delta_{k, \ell}}{(n+1)\left(a_{\ell}+b_{\ell}\right)+q_{\ell}} .
\end{gathered}
$$

Alors

$$
\pi_{k, n}(j+1)=L_{k, k}^{(n)} \pi_{k, n-1}(j)+\sum_{\ell \neq k} M_{k, \ell}^{(n)} \pi_{\ell, n}(j)+N_{k, k}^{(n)} \pi_{k, n+1}(j) .
$$

Donc $\pi(j+1)=H \pi(j)$, où la matrice $H$ a la structure triangulaire par blocs

$$
H=\left(\begin{array}{cccc}
M^{(0)} & N^{(0)} & 0 & \cdots \\
L^{(1)} & M^{(1)} & N^{(1)} & \ddots \\
0 & L^{(2)} & M^{(2)} & \ddots \\
\vdots & \ddots & \ddots & \ddots
\end{array}\right),
$$

comme dans l'équation (1.1) de Gaver et al. (1984). Remarquer au passage que $L^{(1)}=0$. Pour tout $j \geq 0$, on a $\pi(j)=H^{j} \pi(0)$. L'ensemble des états $(k, n)$ 
avec $n=0$ est absorbant. Donc la probabilité d'extinction $\Omega_{k, n}$ partant de $n$ personnes dans l'environnement $k$ est la plus petite solution du système

$$
\Omega=\Omega H, \quad \Omega_{k, 0}=1 \forall k,
$$

où $\Omega$ est le vecteur ligne $\left(\Omega_{k, n}\right)$ avec des indices ordonnés comme avant (Bouleau, 1988, p. 76). Plus explicitement, on a

$$
\Omega_{k, n}=\frac{\Omega_{k, n-1} n b_{k}}{n\left(a_{k}+b_{k}\right)+q_{k}}+\sum_{\ell \neq k} \frac{\Omega_{\ell, n} Q_{\ell, k}}{n\left(a_{k}+b_{k}\right)+q_{k}}+\frac{\Omega_{k, n+1} n a_{k}}{n\left(a_{k}+b_{k}\right)+q_{k}},
$$

qui est équivalent à l'équation (4.1) de Cogburn et Torrez (1981). $\Omega$ peut être calculé numériquement en tronquant les matrices à un ordre suffisamment grand et en prenant la limite quand $i \rightarrow+\infty$ de $\Omega^{(i)}$ avec $\Omega^{(i+1)}=\Omega^{(i)} H$ et $\Omega_{k, n}^{(0)}=\delta_{n, 0}$ pour tout $k$ et tout $n$. Pour les exemples numériques, on a tronqué à $n=500$ et itéré jusqu'à $i=20000$. Avec $a_{1}=2,7$ on obtient $\Omega_{1,1} \simeq \Omega_{2,1} \simeq 1,0$. Avec $a_{1}=5,4$ on obtient $\Omega_{1,1} \simeq 0,61$ et $\Omega_{2,1} \simeq 0,84$, en accord très proche avec la probabilité moyenne d'extinction calculée auparavant.

\subsection{Lien avec l'espérance de la population}

Soit $p(t, n)$ la probabilité que la population soit de taille $n$ au temps $t$. Le seuil pour $\omega$ est le même que celui pour la croissance ou décroissance de l'espérance de la population $\mathcal{E}(t)=\sum_{n \geq 1} n p(t, n)$ pour une histoire environnementale choisie au hasard. En effet, $d \mathcal{E} / d t=(a(t)-b(t)) \mathcal{E}(t)$. Il est clair que $\frac{1}{t} \log \mathcal{E}(t) \rightarrow$ $\left(a_{1}-b_{1}\right) u_{1}+\left(a_{2}-b_{2}\right) u_{2}$ presque sûrement quand $t \rightarrow+\infty$. Cette limite a le même signe que $R_{0}-1$.

Une autre manière de voir cela est de considérer la suite d'environnements $1 \rightarrow 2 \rightarrow 1 \rightarrow 2 \cdots$. Soit $\tau_{n}^{(k)}$ la durée aléatoire passée dans l'environnement $k$ ( $k=1$ ou 2) la $n^{\mathrm{e}}$ fois $(n \geq 1)$. En d'autres termes, l'environnement est d'abord dans l'état 1 pendant $\tau_{1}^{(1)}$ unités de temps, puis dans l'environnement 2 pendant $\tau_{1}^{(2)}$ unités de temps, puis dans l'état 1 pendant $\tau_{2}^{(1)}$ unités de temps, etc. Après $N$ périodes $1 \rightarrow 2$, l'espérance de la population engendrée par un individu est

$$
M_{N}=\exp \left(\sum_{n=1}^{N}\left(a_{1}-b_{1}\right) \tau_{n}^{(1)}+\left(a_{2}-b_{2}\right) \tau_{n}^{(2)}\right)
$$

Donc

$$
\begin{aligned}
\frac{\log M_{N}}{N} & =\left(a_{1}-b_{1}\right) \frac{\sum_{n=1}^{N} \tau_{n}^{(1)}}{N}+\left(a_{2}-b_{2}\right) \frac{\sum_{n=1}^{N} \tau_{n}^{(2)}}{N} \\
& \underset{N \rightarrow+\infty}{\longrightarrow} \frac{a_{1}-b_{1}}{q_{1}}+\frac{a_{2}-b_{2}}{q_{2}}=\frac{\left(a_{1}-b_{1}\right) q_{2}+\left(a_{2}-b_{2}\right) q_{1}}{q_{1} q_{2}}
\end{aligned}
$$

La limite a le même signe que $R_{0}-1$ puisque $u_{1}=q_{2} /\left(q_{1}+q_{2}\right)$ et $u_{2}=$ $q_{1} /\left(q_{1}+q_{2}\right)$. Donc $M_{N}$ tend vers 0 si $R_{0}<1$ et vers $+\infty$ si $R_{0}>1$. 
Noter cependant que ces remarques ne sont pas directement liées à la proposition 1 car elle donne des informations sur l'espérance de la population et non sur la probabilité que cette population s'éteigne. En réalité, pour des processus de branchement en temps discret dans un environnement aléatoire, la population peut très bien être sous-critique et tendre vers l'extinction presque sûrement même si l'espérance de la population tend vers l'infini (Haccou et al., 2005, p. 51).

\subsection{Autres remarques}

Un autre paramètre lié à la croissance d'une espérance. Grav et al. (2012) ont montré que la position de (3) par rapport à 1 sert de seuil entre l'extinction et la persistance pour un modèle épidémique de type SIS constitué d'équations différentielles dans un environnement aléatoire, c'est-à-dire sans stochasticité démographique. Ce nombre $R_{0}$ est appelé $T_{0}$ par Gray et al. (2012) et $\tilde{R}_{0}$ par Britton et Lindholm (2009). Ces deux références utilisent la notation $\ll R_{0} \gg$ pour un nombre différent, à savoir le rayon spectral de (2) dans le cas de deux environnements; appelons le $R^{*}$ pour éviter la confusion. Dans le cas de modèles en temps discret, Bacaër et Khaladi (2012) l'ont appelé $R_{*}$. Comme expliqué ci-dessous, la position de $R^{*}$ par rapport à 1 décide si une certaine espérance croît ou décroît. Voici comment $R^{*}$ est obtenu en général; pour un calcul informel dans le cas particulier où $K=2$, voir la section 3 de Gray et al. (2012). Considérons une fois encore la chaîne de Markov en temps continu sur l'ensemble $\{1,2, \ldots, K\} \times \mathbb{N}$ gouvernée par (6). Soit $E_{k}(t)=\sum_{n \geq 1} n p_{k, n}(t)$ l'espérance. Alors on voit facilement que

$$
\frac{d E_{k}}{d t}=\left(a_{k}-b_{k}\right) E_{k}+\sum_{\ell \neq k}\left(Q_{k, \ell} E_{\ell}-Q_{\ell, k} E_{k}\right) .
$$

Soit $A=\operatorname{diag}\left(a_{1}, \ldots, a_{K}\right), B=\operatorname{diag}\left(b_{1}, \ldots, b_{K}\right)$ et $E(t)=\left(E_{1}(t), \ldots, E_{K}(t)\right)$. Alors $d E / d t=(A-B+Q) E$. En utilisant des résultats standards (Diekmann et al., 2013), on voit que le vecteur d'espérances $E(t)$ tend vers l'infini si et seulement si le rayon spectral $R^{*}$ de $A(B-Q)^{-1}$ est strictement supérieur à 1. Pour l'exemple numérique ci-dessus avec $a_{1}=2,7$, on obtient

$$
R^{*}=\rho\left(A(B-Q)^{-1}\right)=\rho\left(\begin{array}{cc}
\frac{a_{1}\left(b_{2}+q_{2}\right)}{b_{1} b_{2}+q_{2} b_{2}+q_{2} b_{1}} & \frac{a_{1} q_{2}}{b_{1} b_{2}+q_{1} b_{2}+q_{2} b_{1}} \\
\frac{a_{2} q_{1}}{b_{1} b_{2}+q_{1} b_{2}+q_{2} b_{1}} & \frac{a_{2}\left(b_{1}+q_{1}\right)}{b_{1} b_{2}+q_{1} b_{2}+q_{2} b_{1}}
\end{array}\right) \simeq 1,057>1 .
$$

Rappelons que $R_{0}=0,875<1$ et que $\omega=1$ dans ce cas. Donc la position de $R^{*}$ par rapport à 1 décide de la croissance de l'espérance $E(t)$ mais ne donne pas le bon seuil pour l'extinction.

Encore un autre paramètre. Comme déjà noté ci-dessus, la suite des environnements est périodique quand il n'y a que deux environnements possibles : $1 \rightarrow 2 \rightarrow 1 \rightarrow 2 \cdots$. L'espérance de la population engendrée par un individu $t$ unités de temps après que l'environnement a basculé vers l'état $k$ est $e^{\left(a_{k}-b_{k}\right) t}$. 
L'environnement $k$ dure entre $t$ et $t+d t$ unités de temps avec une probabilité $q_{k} e^{-q_{k} t} d t$. Donc l'espérance de la population, $m_{k}$, engendrée par un individu dans l'environnement $k$ ( $k=1$ ou 2$)$ est

$$
m_{k}=\int_{0}^{\infty} q_{k} e^{-q_{k} t} e^{\left(a_{k}-b_{k}\right) t} d t=\frac{q_{k}}{b_{k}+q_{k}-a_{k}}=\frac{1}{1-\frac{a_{k}-b_{k}}{q_{k}}}
$$

pourvu que $b_{k}+q_{k}>a_{k}$, ce qui se trouve être le cas dans l'exemple où $a_{1}=2,7$ : $b_{1}+q_{1}-a_{1}=0,3$ et $b_{2}+q_{2}-a_{2}=2,2$. Noter que $m_{k}$ est infini lorsque $b_{k}+q_{k} \leq a_{k}$. Posons $R_{\star}=m_{1} m_{2}$ (à ne pas confondre avec le $R^{*}$ de la précédente remarque). Alors $R_{\star}=1 / 0,66 \simeq 1,52>1$. Britton et Lindholm (2009, Section 3) ont suggéré que $R_{\star}>1$ était équivalent à $\omega<1$. Mais ici on a $R_{\star}>1$ tandis que $\omega=1$. Donc on voit que la position de $R_{\star}$ par rapport à 1 ne donne pas le bon seuil pour l'extinction. Lorsque $K=2$, le $\S 5.1$ de Britton et Lindholm (2009) montre (avec nos notations) que $R_{\star}>1$ si et seulement si $R^{*}>1$.

\section{$3 \quad$ Les modèles à plusieurs types}

Pour les processus linéaires de naissance et de mort à plusieurs types dans un environnement variable dans le temps, soit $p\left(t, n_{1}, \ldots, n_{m}\right)$ la probabilité d'avoir $n_{i}$ personnes de type $i(1 \leq i \leq m)$ au temps $t$. La fonction génératrice

$$
g\left(t, x_{1}, \ldots, x_{m}\right)=\sum_{n_{1}, \ldots, n_{m} \geq 0} p\left(t, n_{1}, \ldots, n_{m}\right) x_{1}^{n_{1}} \ldots x_{m}^{n_{m}}
$$

vérifie l'équation

$$
\frac{\partial g}{\partial t}=\sum_{i, j}\left[A_{i, j}(t) x_{j}-B_{i, j}(t)\right]\left(x_{i}-1\right) \frac{\partial g}{\partial x_{j}}
$$

(Bacaër et Ait Dads, 2012). La matrice des naissances $A(t)=\left(A_{i, j}(t)\right)_{1 \leq i, j \leq m}$ est à coefficients positifs ou nuls. La matrice des mortalités $B(t)=\left(B_{i, j}(t)\right)$ est de la forme

$$
B_{i, j}(t)=-b_{i, j}(t) \quad \forall i \neq j, \quad B_{j, j}(t)=b_{j, j}(t)+\sum_{i \neq j} b_{i, j}(t) \quad \forall j,
$$

avec $b_{i, j}(t) \geq 0$ pour tout $i$ et $j$ et tout $t$. Supposons que les matrices $(A(t), B(t))$ appartiennent à une liste finie d'environnements $\left(\left(A^{(k)}, B^{(k)}\right)\right)_{1 \leq k \leq K}$, c'est-àdire $A(t)=A^{(\theta(t))}$ et $B(t)=B^{(\theta(t))}$ avec $\theta(t)$ un processus stochastique à valeurs dans $\{1,2, \ldots, K\}$. On suppose encore une fois que les basculements entre les environnements suivent une chaîne de Markov homogène en temps continu. Pour $k \neq \ell$, soit $Q_{k, \ell}$ le taux auquel l'environnement peut basculer de $\ell$ vers $k$. Soit $Q$ la matrice de transition correspondante avec $Q_{\ell, \ell}=-q_{\ell}$ et $q_{\ell}=\sum_{k \neq \ell} Q_{k, \ell}$. Supposons que la matrice $Q$ soit irréductible. Par conséquent, il y a une unique distribution stationnaire $u$ telle que $Q u=0$ et $\sum_{k} u_{k}=1$. Enfin on suppose 
que $b_{j, j}^{(k)}>0$ pour tout $k$ et $j$. Cette hypothèse implique que le plus grand exposant de Lyapunov du système différentiel aléatoire $d Z / d t=-B(t) Z(t)$ est strictement négatif.

Au temps $t=0$, supposons qu'il y ait $\nu_{i}$ personnes de type $i$, avec $\nu_{i} \in \mathbb{N}$. Supposons de plus qu'il existe $i$ tel que $\nu_{i}>0$. Alors

$$
g\left(0, x_{1}, \ldots, x_{m}\right)=x_{1}^{\nu_{1}} \cdots x_{m}^{\nu_{m}} .
$$

L'objectif est de calculer la probabilité d'extinction $\omega$, qui est la limite quand $t \rightarrow$ $+\infty$ de $p(t, 0, \ldots, 0)$, c'est-à-dire de $g(t, 0, \ldots, 0)$. C'est une variable aléatoire qui dépend de l'histoire environnementale.

Comme l'expliquent par exemple Bacaër et Ait Dads (2012), $\omega$ peut être calculé en utilisant les caractéristiques de (8). Pour tout $\tau \geq 0$, soit $Y^{(\tau)}$ la solution unique du système

$$
\frac{d Y_{j}^{(\tau)}}{d s}(s)=\sum_{i}\left[A_{i, j}(-s)\left(1-Y_{j}^{(\tau)}(s)\right)-B_{i, j}(-s)\right] Y_{i}^{(\tau)}(s)
$$

avec la condition initiale $Y_{j}^{(\tau)}(-\tau)=1$ pour tout $j$. Alors

$$
\omega=\left(\omega_{1}\right)^{\nu_{1}} \cdots\left(\omega_{m}\right)^{\nu_{m}} \quad \text { et } \quad \omega_{j}=1-\lim _{\tau \rightarrow+\infty} Y_{j}^{(\tau)}(0) .
$$

La question est de savoir si $\omega=1$ ou $\omega<1$. Le résultat dépend de la stabilité du système d'équations différentielles aléatoires (Arnold, 1998, Sect. 2.2)

$$
\frac{d X}{d t}=(A(t)-B(t)) X(t)
$$

qui est l'équation satisfaite par le vecteur des espérances des populations au temps $t$. Cette stabilité dépend du signe de $\lambda_{1}(A, B)$, le plus grand exposant de Lyapunov de (12). En suivant Bacaër et Khaladi (2012), la stabilité peut alternativement être formulée en termes de reproductivité nette $R_{0}$, qui est l'unique solution de

$$
\lambda_{1}\left(A / R_{0}, B\right)=0 .
$$

Une manière d'étudier la probabilité d'extinction $\omega$ serait d'adapter la méthode utilisée par Bacaër et Ait Dads (2012) pour des environnements périodiques au cas des environnements aléatoires, en prenant avantage du fait que le système (11) est coopératif et sous-homogène comme dans les travaux de Chueshov (2002) ou Benaïm et Schreiber (2009). Ceci conduirait à des difficultés techniques telles que le lien entre $\lambda_{1}(A, B)$ et le plus grand exposant de Lyapunov de la linéarisation près de zéro de (11), qui est l'adjoint de (12); voir Arnold et Wihstutz (1986) ou Barreira et Valls (2008). La preuve de la persistance de (11) quand $R_{0}>1$ peut aussi être difficile. Pour éviter ces difficultés, on va utiliser la même idée que dans la seconde preuve de la proposition 11: pour un nombre fini d'environnements markoviens, le problème en temps continu peut être ramené à un processus de branchement à plusieurs types en temps discret dans un environnement aléatoire. Les résultats d Athreva et Karlin (1971) peuvent alors être appliqués. 
Proposition 2. Supposons que la matrice $C^{(k)}:=A^{(k)}-B^{(k)}$ soit irréductible pour tout $k$. Supposons en plus que pour tout $k$, il existe $(i, j)$ tel que $A_{i, j}^{(k)}>0$. Si $R_{0} \leq 1$, alors $\omega=1$ presque sûrement. Si $R_{0}>1$, alors $\omega<1$ presque sûrement.

Démonstration. Posons $t_{0}=0$. Soient $\left(t_{n}\right)_{n \geq 1}$ avec $0<t_{1}<t_{2}<\cdots$ les temps auxquels les environnements basculent. Pour tout $n \geq 0$, soit $k_{n}\left(1 \leq k_{n} \leq K\right)$ l'environnement dans l'intervalle de temps $\left(t_{n}, t_{n+1}\right)$. Dans l'environnement $k$, un individu de type $h$ au départ engendre une population $t$ unités de temps plus tard dont la fonction génératrice $\phi^{(k, h)}\left(t, x_{1}, \ldots, x_{m}\right)$ vérifie

$$
\frac{\partial \phi^{(k, h)}}{\partial t}=\sum_{i, j}\left[A_{i, j}^{(k)} x_{j}-B_{i, j}^{(k)}\right]\left(x_{i}-1\right) \frac{\partial \phi^{(k, h)}}{\partial x_{j}}
$$

pour $t>0$ et $\left(x_{1}, \ldots, x_{m}\right) \in(0,1)^{m}$ tandis que $\phi^{(k, h)}\left(0, x_{1}, \ldots, x_{m}\right)=x_{h}$. Posons

$$
M_{i}^{(k, h)}(t)=\frac{\partial \phi^{(k, h)}}{\partial x_{i}}(t, 1, \ldots, 1), \quad M^{(k)}(t)=\left(M_{i}^{(k, h)}(t)\right)_{i, h} .
$$

Alors $M_{i}^{(k, h)}(t)$ est l'espérance de la population de type $i$. En partant de (14) ou en se référant à Athreya et Ney (1972), on voit (cf. appendice) que

$$
\frac{d M^{(k)}}{d t}(t)=C^{(k)} M^{(k)}(t)
$$

pour tout $t>0$ tandis que $M^{(k)}(0)=I$ (la matrice identité). Par conséquent,

$$
\mathcal{M}_{n}:=M^{\left(k_{n}\right)}\left(t_{n+1}-t_{n}\right)=\exp \left[C^{\left(k_{n}\right)}\left(t_{n+1}-t_{n}\right)\right] .
$$

Noter avec (12) et (16) que 1

$$
\lambda_{1}(A, B)=\lim _{n \rightarrow+\infty} \frac{1}{n} \log \left\|\mathcal{M}_{n-1} \mathcal{M}_{n-2} \cdots \mathcal{M}_{0}\right\|
$$

presque sûrement. D'après Athreya et Karlin (1971, Théorème 12), le signe de cette limite décide s'il y a extinction presque sûrement ou pas. Mais d'abord il faut vérifier les trois hypothèses de ce théorème. L'irréductibilité de $C^{\left(k_{n}\right)}$ implique que tous les coefficients de $\mathcal{M}_{n}$ sont strictement positifs (Berman et Plemmons, 1994, Théorème 6.3.12) : la première hypothèse est satisfaite. Posons maintenant

$$
S_{i, j}^{(k, h)}(t)=\frac{\partial^{2} \phi^{(k, h)}}{\partial x_{i} \partial x_{j}}(t, 1, \ldots, 1), \quad S^{(k, h)}(t)=\left(S_{i, j}^{(k, h)}(t)\right)_{i, j} .
$$

\footnotetext{
1. Erratum : $1 / n$ doit être remplacé par $1 / t_{n}$, ce qui ne change rien à la conclusion puisque $t_{n} / n$ tend vers une limite positive.
} 
On peut montrer que tous les coefficients de la matrice $S^{\left(k_{n}, h\right)}\left(t_{n+1}-t_{n}\right)$ sont aussi strictement positifs (cf. appendice) : c'est la seconde hypothèse. Enfin on a aussi

$$
-\sum_{k} \int_{0}^{\infty} w_{k, t} \log \left[\sum_{h=1}^{m}\left(1-\phi^{(k, h)}(t, 0, \ldots, 0)\right)\right] d t<+\infty
$$

où $w_{k, t}$ est donné par (5), à cause de la décroissance exponentielle de $w_{k, t}$ quand $t \rightarrow+\infty$ et puisque $1-\phi^{(k, h)}(t, 0, \ldots, 0)$ ne peut s'approcher de 0 plus vite que $e^{-c t}$ pour un $c>0$ [ce $c$ est donné par le taux auquel la solution de (11) peut s'approcher de 0 dans un environnement $k$ qui est sous-critique]. Donc la troisième condition est aussi satisfaite.

Si $R_{0} \leq 1$, alors $\lambda_{1}(A, B) \leq 0$. On conclut avec Athreya et Karlin (1971, Théorème 12(i)) quand $\lambda_{1}(A, B)<0$ ou avec Kaplan (1974, Théorème 2) quand $\lambda_{1}(A, B)=0$ que $\omega=1$ presque sûrement.

Si $R_{0}>1$, alors $\lambda_{1}(A, B)>0$. On conclut avec Athreya et Karlin (1971, Théorème $12($ ii) ) que $\omega<1$.

Exemple. Considérons comme Bacaër et Ait Dads (2012) un modèle épidémique SEIR linéarisé, c'est-à-dire un processus de naissance et de mort à deux types, mais supposons que l'environnement varie aléatoirement entre deux états. Supposons que la matrice de transition soit constante :

$$
Q=\left(\begin{array}{cc}
-q_{1} & q_{2} \\
q_{1} & -q_{2}
\end{array}\right)
$$

avec $q_{1}>0$ et $q_{2}>0$. La distribution stationnaire est telle que $u_{1}=q_{2} /\left(q_{1}+q_{2}\right)$ et $u_{2}=q_{1} /\left(q_{1}+q_{2}\right)$. Supposons que

$$
A(t)=\left(\begin{array}{cc}
0 & \beta(t) \\
0 & 0
\end{array}\right), \quad B(t)=\left(\begin{array}{cc}
\alpha+\mu & 0 \\
-\alpha & \gamma+\mu
\end{array}\right),
$$

où le taux de contact effectif $\beta(t)$ est égal à $\beta_{1}>0$ ou à $\beta_{2}>0$ selon l'environnement, $\alpha>0$ est le taux auquel les gens infectés mais pas encore infectieux deviennent infectieux, $\mu>0$ est la mortalité et $\gamma>0$ est le taux de guérison. La reproductivité nette $R_{0}$ est l'unique nombre positif tel que le plus grand exposant de Lyapunov du système $d X / d t=\left(A(t) / R_{0}-B(t)\right) X(t)$ soit égal à 0 . Noter que si $\beta(t)$ était constant et égal à sa moyenne temporelle $u_{1} \beta_{1}+u_{2} \beta_{2}$, on aurait $R_{0}=\left(u_{1} \beta_{1}+u_{2} \beta_{2}\right) \alpha /((\alpha+\mu)(\gamma+\mu))$. Des formules analytiques approchées pour $R_{0}$ dans un environnement aléatoire peuvent être déduites des formules pour le plus grand exposant de Lyapunov des systèmes bidimensionnels données par Arnold et Kloeden (1989).

La probabilité pour que le processus soit éteint au temps $\tau>0$ partant de $\left(E_{0}, I_{0}\right)$ personnes au temps 0 est $\left(1-Y_{1}^{(\tau)}(0)\right)^{E_{0}}\left(1-Y_{2}^{(\tau)}(0)\right)^{I_{0}}$, avec pour tout 
$-\tau<s<0$

$$
\begin{aligned}
& \frac{d Y_{1}^{(\tau)}}{d s}(s)=-(\alpha+\mu) Y_{1}^{(\tau)}(s)+\alpha Y_{2}^{(\tau)}(s), \\
& \frac{d Y_{2}^{(\tau)}}{d s}(s)=\beta(-s) Y_{1}^{(\tau)}(s)\left(1-Y_{2}^{(\tau)}(s)\right)-(\gamma+\mu) Y_{2}^{(\tau)}(s),
\end{aligned}
$$

tandis que $Y_{1}^{(\tau)}(-\tau)=1$ et $Y_{2}^{(\tau)}(-\tau)=1$. Il y a des erreurs de signes dans les équations correspondantes données par Bacaër et Ait Dads (2012) ; les figures 3 et 4 dans cette référence sont néanmoins correctes. Soient $\omega_{1}$ et $\omega_{2}$ les probabilités d'extinction ultimes partant soit d'une personne infectée mais non infectieuse, soit d'une personne infectieuse $: \omega_{j}=\lim _{\tau \rightarrow+\infty} 1-Y_{j}^{(\tau)}(0)$ pour $j=1,2$. La proposition 2 montre que $\omega_{1}=\omega_{2}=1$ presque sûrement si $R_{0} \leq 1$ et que $\omega_{1}<1$ et $\omega_{2}<1$ presque sûrement si $R_{0}>1$.

Si $\beta_{2} \leq \beta_{1}$ alors un théorème de comparaison pour le système (19)-(20) montre que $\omega_{1}$ et $\omega_{2}$ sont supérieures aux probabilités correspondantes pour le processus de naissance et de mort où l'environnement est toujours 1 . Si ce dernier processus est surcritique alors ces probabilités (appelons les $\xi_{1}$ et $\xi_{2}$ ) se calculent facilement soit en déterminant l'état stationnaire de (19)-(20) avec $\beta(-s)$ remplacé par $\beta_{1}$, soit en considérant le processus de Bienaymé-GaltonWatson à plusieurs types induit : $\xi_{1}=\frac{\mu}{\alpha+\mu}+\frac{\gamma+\mu}{\beta_{1}}$ et $\xi_{2}=\frac{\alpha+\mu}{\alpha} \frac{\gamma+\mu}{\beta_{1}}$.

Prenons $q_{1}=q_{2}=1, \beta_{1}=2, \beta_{2}=1, \alpha=1, \mu=0,01$ et $\gamma=1$. On obtient $R_{0} \simeq 1,45>1$. Noter que pour le système moyenné en temps, on a $R_{0} \simeq$ 1,47. De plus, on obtient $\xi_{1} \simeq \xi_{2} \simeq 0,51$. La figure 2 montre l'histogramme pour la probabilité d'extinction partant d'une personne infectée mais non infectieuse dans l'environnement 1 . Il a été obtenu avec 1000 simulations de l'histoire environnementale. La moyenne est proche de 0,69 (elle serait 0,66 partant de l'environnement 2). On a pris $\tau=100$ et un pas de temps de 0,001. La figure tend à confirmer que $R_{0}>1$ implique $\omega_{1}<1\left(\right.$ et $\left.\omega_{2}<1\right)$ presque sûrement.

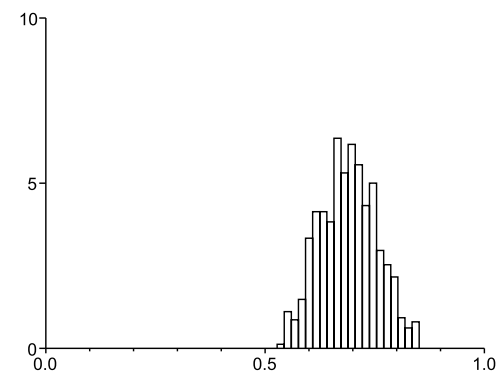

Figure 2 - Histogramme de la probabilité d'extinction $\omega_{1}$ partant d'une personne infectée mais non infectieuse dans l'environnement 1 . 


\section{Conclusion}

Certaines questions restent ouvertes. On peut se demander, comme Britton et Lindholm (2009), ce qui arrive si la survie n'est pas distribuée exponentiellement, c'està-dire pour des processus de Crump-Mode-Jagers tels que dans le travail de Ball et Donnelly (1995). Il est aussi clair que la plupart de résultats restent vrais pas seulement pour un nombre fini d'environnements markoviens mais aussi pour des environnements ergodiques plus généraux.

Pour les applications biologiques, il serait plus réaliste de supposer que la matrice de transition est de la forme

$$
Q(t)=\left(\begin{array}{cc}
-q_{1}(t) & q_{2}(t) \\
q_{1}(t) & -q_{2}(t)
\end{array}\right),
$$

où par exemple $q_{1}(t)=k_{1}\left(1+\varepsilon_{1} \cos \omega t\right), q_{2}(t)=k_{2}\left(1+\varepsilon_{2} \sin \omega t\right), k_{1}>0$, $k_{2}>0, \varepsilon_{1} \in(0,1)$ et $\varepsilon_{2} \in(0,1)$. De cette manière, l'année est plus ou moins divisée en deux saisons (disons l'été et l'hiver), l'une qui serait favorable à la croissance et l'autre moins favorable. Cela pourrait être le cas pour les maladies infectieuses où le taux effectif de contact dépend de la température. Une matrice $Q(t)$ périodique comme ci-dessus est un modèle de saisonnalité plus réaliste qu'une matrice $Q$ constante. Dans ce dernier cas avec seulement deux environnements, les deux saisons alternent mais les longueurs des saisons sont indépendantes. Pour être réaliste il faut qu'un été particulièrement court soit suivi d'un hiver particulièrement long pour garder plus ou moins la périodicité annuelle.

Remerciements On remercie le professeur Khaladi pour ses encouragements. On remercie aussi les professeurs Ball et Bansaye et particulièrement le professeur Jagers pour leurs commentaires sur certaines parties de ce travail.

\section{Appendice}

En prenant la dérivée de (14) par rapport à $x_{I}$, on obtient

$$
\begin{aligned}
\frac{\partial^{2} \phi^{(k, h)}}{\partial t \partial x_{I}}= & \sum_{i, j}\left[A_{i, j}^{(k)} \delta_{j, I}\left(x_{i}-1\right)+\left(A_{i, j}^{(k)} x_{j}-B_{i, j}^{(k)}\right) \delta_{i, I}\right] \frac{\partial \phi^{(k, h)}}{\partial x_{j}} \\
& +\sum_{i, j}\left(A_{i, j}^{\left(k_{n}\right)} x_{j}-B_{i, j}^{\left(k_{n}\right)}\right)\left(x_{i}-1\right) \frac{\partial^{2} \phi^{(k, h)}}{\partial x_{I} \partial x_{j}},
\end{aligned}
$$

où $\delta$ est le symbole de Kronecker. En prenant $x_{1}=\cdots=x_{m}=1$, on obtient

$$
\frac{\partial}{\partial t}\left[\frac{\partial \phi^{(k, h)}}{\partial x_{I}}(t, 1, \ldots, 1)\right]=\sum_{j}\left(A_{I, j}^{(k)}-B_{I, j}^{(k)}\right) \frac{\partial \phi^{(k, h)}}{\partial x_{j}}(t, 1, \ldots, 1) .
$$


Ceci est identique à (16). En prenant la dérivée par rapport à $x_{J}$ de (21) et en prenant une nouvelle fois $x_{1}=\cdots=x_{m}=1$, on obtient aussi que

$$
\begin{aligned}
\frac{\partial}{\partial t}\left[\frac{\partial^{2} \phi^{(k, h)}}{\partial x_{I} \partial x_{J}}(t, 1, \ldots, 1)\right]= & A_{J, I}^{(k)} \frac{\partial \phi^{(k, h)}}{\partial x_{I}}(t, 1, \ldots, 1) \\
& +A_{I, J}^{(k)} \frac{\partial \phi^{(k, h)}}{\partial x_{J}}(t, 1, \ldots, 1) \\
& +\sum_{j}\left(A_{I, j}^{(k)}-B_{I, j}^{(k)}\right) \frac{\partial^{2} \phi^{(k, h)}}{\partial x_{j} \partial x_{J}}(t, 1, \ldots, 1) \\
& +\sum_{j}\left(A_{J, j}^{(k)}-B_{J, j}^{(k)}\right) \frac{\partial^{2} \phi^{(k, h)}}{\partial x_{I} \partial x_{j}}(t, 1, \ldots, 1) .
\end{aligned}
$$

Un calcul semblable se trouve par exemple dans le $\S$ V.7.3 du livre d Athreya et Ney (1972). Rappelons les notations (15) et (18) pour les dérivées premières et secondes. Posons

$$
G_{i, j}^{(k, h)}(t)=A_{i, j}^{(k)} M_{j}^{(k, h)}(t)+A_{j, i}^{(k)} M_{i}^{(k, h)}(t), \quad G^{(k, h)}(t)=\left(G_{i, j}^{(k, h)}(t)\right)_{i, j} .
$$

Alors (22) est identique à

$$
\frac{d S_{I, J}^{(k, h)}}{d t}(t)=\sum_{j}\left[C_{I, j}^{(k)} S_{j, J}^{(k, h)}(t)+C_{J, j}^{(k)} S_{j, I}^{(k, h)}(t)\right]+G_{I, J}^{(k, h)}(t)
$$

pour $t>0$. En utilisant la symétrie de la matrice $S^{(k, h)}(t)$, cette équation s'écrit

$$
\frac{d S^{(k, h)}}{d t}(t)=C^{(k)} S^{(k, h)}(t)+S^{(k, h)}(t)\left(C^{(k)}\right)^{*}+G^{(k, h)}(t),
$$

où * désigne la matrice transposée. Mais $\phi^{(k, h)}\left(0, x_{1}, \ldots, x_{m}\right)=x_{h}$ implique que $S^{(k, h)}(0)$ est la matrice nulle. Comme dans le livre d Athreya et Ney (1972, formule (15), p. 203), on obtient

$$
S^{(k, h)}(t)=\int_{0}^{t} e^{(t-u) C^{(k)}} G^{(k, h)}(u)\left(e^{(t-u) C^{(k)}}\right)^{*} d u
$$

pour tout $t \geq 0$. Puisque $M_{i}^{(k, h)}(t)>0$ pour tout $i$ et tout $t>0$, et puisque par hypothèse il existe $(i, j)$ tel que $A_{i, j}^{(k)}>0$, on voit que la matrice à coefficients positifs ou nuls $G^{(k, h)}(t)$ a au moins un coefficient strictement positif pour tout $t>0$. Comme tous les coefficients de la matrice $e^{(t-u) C^{(k)}}$ et de sa transposée sont strictement positifs lorsque $u<t$, on voit que tous les coefficients de la matrice sous l'integrale de (23) sont strictement positifs pour $u<t$. Donc tous les coefficients de la matrice $S^{(k, h)}(t)$ sont aussi strictement positifs pour $t>0$. 


\section{Références}

Arnold L (1998) Random dynamical systems. Springer, Berlin

Arnold L, Kloeden P (1989) Lyapunov exponents and rotation number of twodimensional systems with telegraphic noise. SIAM J Appl Math 49 :1242-1274

Arnold L, Wihstutz V (1986) Lyapunov exponents : a survey. In : Arnold L, Wihstutz V (eds) Lyapunov exponents, Lecture Notes in Mathematics 1186, Springer, Berlin, pp 1-26

Artalejo JR, Economou A, Lopez-Herrero MJ (2012) Stochastic epidemic models with random environment : quasi-stationarity, extinction and final size. J Math Biol, doi :10.1007/s00285-012-0570-5

Athreya KB, Karlin S (1971) On branching processes with random environments : I Extinction probabilities. Ann Math Stat 42 :1499-1520

Athreya KB, Ney PE (1972) Branching processes. Springer, Berlin

Bacaër N, Ait Dads E (2012) On the probability of extinction in a periodic environment. J Math Biol, doi :10.1007/s00285-012-0623-9

Bacaër N, Guernaoui S (2006) The epidemic threshold of vector-borne diseases with seasonality. J Math Biol 53 :421-436

Bacaër N, Khaladi M (2012) On the basic reproduction number in a random environment. J Math Biol, doi :10.1007/s00285-012-0611-0

Ball F, Donnelly P (1995) Strong approximations for epidemic models. Stoch Proc Applic $55: 1-21$

Barreira L, Valls C (2008) Stability of nonautonomous differential equations. Springer, Berlin

Benaïm M, Schreiber SJ (2009) Persistence of structured populations in random environments. Theoret Popul Biol 76 :19-34

Berman A, Plemmons RJ (1994) Nonnegative matrices in the mathematical sciences. SIAM, Philadelphia

Bouleau N (1988) Processus stochastiques et applications. Hermann, Paris

Britton T, Lindholm M (2009) The early stage behaviour of a stochastic SIR epidemic with term-time forcing. J Appl Probab 46 :975-992

Chueshov I (2002) Monotone random systems. Springer, Berlin

Cogburn R, Torrez WC (1981) Birth and death processes with random environments in continuous time. J Appl Probab $18: 19-30$ 
Diekmann O, Heesterbeek H, Britton T (2013) Mathematical tools for understanding infectious disease dynamics. Princeton University Press, Princeton

Dublin LI, Lotka AJ (1925) On the true rate of natural increase. J Am Stat Assoc 20(151) :305-339

Gaver DP, Jacobs PA, Latouche G (1984) Birth-and-death models in randomly changing environments. Adv Appl Probab 16 :715-731

Gray A, Greenhalgh D, Mao X, Pan J (2012) The SIS epidemic model with Markovian switching. J Math Anal Appl 394 :496-516

Haccou P, Jagers P, Vatutin VA (2005) Branching processes : variation, growth, and extinction of populations. Cambridge University Press, Cambridge

Hernandez-Suarez C, Rabinovich J, Hernandez K (2012) The long-run distribution of births across environments under environmental stochasticity and its use in the calculation of unconditional life-history parameters. Theor Popul Biol 82 :264-274

Hillion A (1986) Les théories mathématiques des populations. Presses Universitaires de France, Paris

Kaplan N (1973) A continuous time Markov branching model with random environments. Adv Appl Probab 5 :37-54

Kaplan N (1974) Some results about multidimensional branching processes with random environments. Ann Probab 2 :441-455

Kendall DG (1948) On the generalised 'birth-and-death' process. Ann Math Statist $19: 1-15$

Lewontin RC, Cohen D (1969) On population growth in a randomly varying environment. Proc Natl Acad Sci USA 62 :10561060

Meyn S, Tweedie R (1993) Markov chains and stochastic stability. Springer, Berlin

Pardoux E (2008) Markov processes and applications. Wiley, Chichester

Yechiali U (1973) A queuing-type birth-and-death process defined on a continuous-time Markov chain. Operations Research 21 :604-609 